\title{
Computed Tomography Image Quality Evaluation for pre-surgical dental implant site assessment using Different Exposure Setting Protocols: Mandibular Phantom Study
}

\author{
Ahmed Al-Humairi ${ }^{a^{*}}$, Xiaoming Zheng ${ }^{\text {a }}$, Ryan H.L. Ip ${ }^{\text {a }}$, Bilal El Masoud ${ }^{\text {b,c }}$ \\ ${ }^{a}$ Charles Sturt University, Wagga Wagga, NSW, Australia. \\ ${ }^{\mathrm{b}}$ Charles Sturt University, Orange, NSW, Australia. \\ ${ }^{\mathrm{c}}$ Jordan University of Science and Technology, Irbid. Jordan. \\ *Ahmed Al-Humairi: aali@csu.edu.au
}

\begin{abstract}
The purpose of this study was to compare the effect of various radiographic exposure settings of multi-slices CT (MSCT) scanners on the image quality of several anatomical landmarks used for per-surgical assessment of dental implants. A dry adult skull embedded in plexi material was used in the image acquisitions using both a GE 16 slices CT scanner (Milwaukee, USA) and a Toshiba Aquilion CT scanner (Toshiba Corporation, Japan). The exposure settings were manually adjusted for a full clear image of the head. The image qualities were assessed blindly by three dental implant specialists in randomised order. The image quality score was based on a 5 points scale where 1 is considered as not clearly visible and 5 positively clearly visible and the overall image quality (OIQ) based on 3 point ranking system scale (poor, acceptable and clear). Toshiba MSCT with 200 and $250 \mathrm{~mA}$ produced consistent good image quality in all three tube voltages $(80,100,120 \mathrm{kVp})$ whilst $100 \mathrm{~mA}$ setting provided the lowest image quality. In comparison, the GE MSCT with a $120 \mathrm{~mA}$ has shown acceptable image quality with various tube voltages while good image quality was present consistently with high $\mathrm{kVp}(120)$ and $\mathrm{mA}(120,180$ and 240) settings.
\end{abstract}

Keywords: image quality, MSCT, dose reduction, exposure setting

\section{Introduction}

In the last decade, there has been widespread use of digital two-dimensional (2D) intraoral and panoramic radiography by dentists. However, 2D radiographic images are difficult to interpret due to overlapping of complex osseous structures, with anatomical structures such as the lingual foramen, mental foramen and incisive canal which contain neuro-vascular structures cannot be easily defined. Moreover, the lingual cortical bone of the mandible and the width of the alveolar ridge cannot be accurately examined. Three-dimensional (3D) assessment of craniofacial structures was introduced through development of computed tomography (CT) which has become an ample means for head and neck diagnosis.

Though, CT remains as not yet ideal for the specific diagnostic tool in dental applications, including impacted teeth or apical lesions. Extreme radiation exposure, increased cost and limited availability impede the routine use of this technology for dental application. Nonetheless, Cone Beam CT (CBCT) proposes a good alternative approach but with slight reduction in image quality ${ }^{1,2,3}$.

In recent years, a considerable number of various computer-assisted guided implant systems have been available in clinical practice ${ }^{4}$. One of the most crucial indications of these systems is the clinical factors, such as fabricating the surgical guide and planning drilling angulations. Otherwise, any under-planning based on these factors will lead to clinical errors which will hence govern the success of the outcomes ${ }^{5}$. Therefore, the accuracy and clarity of the obtained 3D image forms the basis upon which many of the computer-assisted implant placement procedures.

There is a relative association between dose and other factors such as tube current, exposure duration and degree of the gantry rotation ${ }^{6}$. Altering to reduce any of these parameters alone or in combination has the capacity to 
decrease patient exposure.

Significant dose reductions may be reached through recognising the clinical indication of the image acquisition, individual features, imaging requirements and parameters of each case. The European Academy of Dentomaxillofacial Radiology, SEDENTEXCT, and recently the Health Protection Agency have established guidelines in utilising CBCT that promote the principle of dose optimisation ${ }^{7,8}$.

The ICRP international system for Radiation protection in medical imaging is based on three critical principles; justification; minimization (ALARA) and limits ${ }^{9,10}$. The first two principles involve the individuals whom are exposed to radiation, while the low dose level application addresses the occupational; and environmental exposures.

The idea of justification based on the exposure should result in benefits that outweigh harm. In this principle the clinical type of scan and field of the scan fall within the justification principles. The clinician refers the patient for radiographic examination while the radiographer is responsible for acquisition of the images. So both patient's referring physician and radiologist should be involved in the justification principle. The principle of optimisation will take place when the justification was achieved.

Low dose protocols in dentistry have been investigated and researched with different approaches. Optimisation protocols was developed for examination of the anterior maxilla in children by optimisation the exposure dose and the clinical and physical image quality ${ }^{11}$. On other retrospective study, by evaluating the image quality for pre-implant placement surgery, there was a significant dose reduction while the diagnostic value of the examination was maintained by using $\mathrm{CBCT}^{8}$. Therefore with $\mathrm{CT}$, scanning may reflect the same conclusion.

As low as reasonably achievable is one of the key goals of the optimization strategies which provoked by ICPR, these strategies protect the patient from any possibility of unnecessary radiation which prevent any deterministic effect of the radiation ${ }^{12,13}$. It is in addition, vital for optimization to address image quality. The image quality, in particular must not be compromised through optimization of dose as these are necessary to aid the physicians in diagnosing diseases and conditions.
The radiologic technologist holds responsibility of dose optimization in the clinical practice. Matthews and Brennan have stated that the individual carrying out the examination holds complete responsibility in optimal technique application, while the technologist controls dose optimization. Furthermore, scope of practice for all CT technologists is based on applying the ALARA principles in ensuring exposure to patient, self and others is kept to a minimum ${ }^{14}$.

The purpose of this study was to compare the effect of various radiographic exposure settings of a mutlisliced CT (MSCT) on the image quality of several anatomical landmarks used for per-surgical assessment of dental implants.

\section{Methodology}

This in vitro study was performed following the ethics and radiation safety committee approvals were obtained from Charles Sturt University (ethic committee and radiation safety board). A skull phantom was utilised, the phantom consists of a dry adult skull embedded in plexi material simulating the soft tissue. The images were then captured with GE (Light-Speed 16, General Electric Medical System, Milwaukee, WI, USA) is equipped with 16 detector rows and has a minimal rotation time of $0.5 \mathrm{~s}$ given a collimation ranging from 0.75 to $1.5 \mathrm{~mm}$ and Toshiba Aquilion ${ }^{\circledR}$ (Toshiba Corporation, Japan) scanner with 16 detector rows, $0.5-\mathrm{mm}$ multislice acquisition (Figure $1)$.

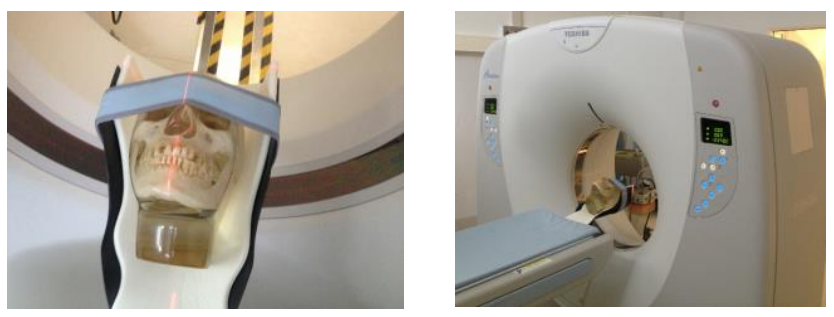

Figure 1. Dry skull phantom, placed in reproducible position in Toshiba MSCT 
This allows rapid scanning of large body segments in relatively short scanning times. The exposure settings were manually adjusted for a full clear image of the head. All the images were taken under the same positioning and field of view. The exposure settings for each machine were operated as mentioned in Table 1.

Table 1. Exposure settings with GE and Toshiba 16 MSCT

\begin{tabular}{|c|c|c|c|c|c|c|}
\hline & \multicolumn{9}{|l|}{ GE } & \multicolumn{3}{l|}{ Toshiba } \\
\hline $\mathbf{k V p}$ & 80 & 100 & 120 & 80 & 100 & 120 \\
\hline \multirow{4}{*}{$\mathbf{m A}$} & 10 & 10 & 10 & 50 & 100 & 100 \\
\cline { 2 - 7 } & 20 & 20 & 20 & 100 & 150 & 150 \\
\cline { 2 - 7 } & 40 & 40 & 40 & 150 & 200 & 200 \\
\cline { 2 - 7 } & 60 & 60 & 60 & 200 & 250 & 250 \\
\cline { 2 - 7 } & 80 & 80 & 80 & 250 & 300 & 300 \\
\cline { 2 - 7 } & 120 & 120 & 120 & & & \\
\cline { 2 - 7 } & 180 & 180 & 180 & & & \\
\cline { 2 - 7 } & 240 & 240 & 240 & & & \\
\cline { 2 - 7 } & 320 & 320 & 320 & & & \\
\cline { 2 - 7 } & 380 & 380 & 380 & & & \\
\hline
\end{tabular}

Data acquisition, planning of examinations, saving images and standardized multiplanar reconstructions were performed by registered radiographers. The images were presented for observation blindly by several dental implant specialists in randomised order. The observers evaluated the visibility of specific anatomical landmarks in each image and the overall image quality as an indication for implant dentistry.

The anatomical landmarks were presented in the mandibles which are relevant to implant placement. The observers scored these anatomical landmarks which have been defined in Table 2. The score was based on a 5 points scale where 1 is considered as not clearly visible and 5 positively clearly visible (ALQ). In addition, the observers ranked the overall image quality (OIQ) in relation to pre-surgical implant placement based on 3 point ranking system scale (poor, acceptable and clear).

During the observation and evaluation process, the observers were asked to replicate the clinical set up such as using glasses or any screen filters. In addition, the observers were instructed to rest their eyes if their eyes experienced strain.
Table 2. Anatomical landmarks definitions

\begin{tabular}{|l|l|}
\hline $\begin{array}{l}\text { Anatomical } \\
\text { landmark }\end{array}$ & Definition \\
\hline Bone width & $\begin{array}{l}\text { Assess the adequacy of depiction of bone width in } \\
\text { different views. }\end{array}$ \\
\hline Bone height & $\begin{array}{l}\text { The height of available bone for implant insertion } \\
\text { with reference to the proximity of relative } \\
\text { anatomical structure }\end{array}$ \\
\hline Inferior & $\begin{array}{l}\text { Will be evaluated by the visibility and the outline } \\
\text { of the canal in the mandibular molar area }\end{array}$ \\
\hline Mental foramen & $\begin{array}{l}\text { Will be evaluated by the visibility of the foramen } \\
\text { in the image }\end{array}$ \\
\hline Lingual Foramen & Will be evaluated of its extent and the visibility \\
\hline $\begin{array}{l}\text { Cortical and } \\
\text { Trabecular Bone }\end{array}$ & \begin{tabular}{l} 
Will be evaluated subjectively \\
\hline
\end{tabular} \\
\hline
\end{tabular}

Intra-examiner reliability was assessed through duplicating some of the images that were presented randomly with others; the inter-examiner reliability was measured by comparing the rating scores between observers.

\section{Results}

The inter-observer agreement was moderate while the intra-observer reliability using weighted Kappa statistics were high with $\mathrm{GE}(\mathrm{O} 1=0.513, \mathrm{O} 2=0.885, \mathrm{O} 3=0.864)$ and Toshiba $(\mathrm{O} 1=0.651, \mathrm{O} 2=0.836, \mathrm{O} 3=0.781)$ devices with respect to all the specific evaluation criteria. Figure 3 describes the relationship between tube current and image quality of the anatomical landmarks, where the image quality would be defined as good, acceptable and unacceptable. The relation of the image quality in relation to various tube voltages was also shown in the graph. Within the present in-vitro study, alterations of exposure settings play an important role in image quality in both MSCT (Figure.2). Through utilising the Toshiba MSCT, the 200, $250 \mathrm{~mA}$ had produced consistent good image quality in all three tube voltage ranges which are $80,100,120 \mathrm{kVp}$, while the $100 \mathrm{~mA}$ setting provided the lowest image quality. In comparison, the GE MSCT, $120 \mathrm{~mA}$ has shown acceptable image quality with various tube voltages, while the good image quality was present consistently with high $\mathrm{kVp}$ (120) 
and $\mathrm{mA}(120,180$ and 240$)$ settings.
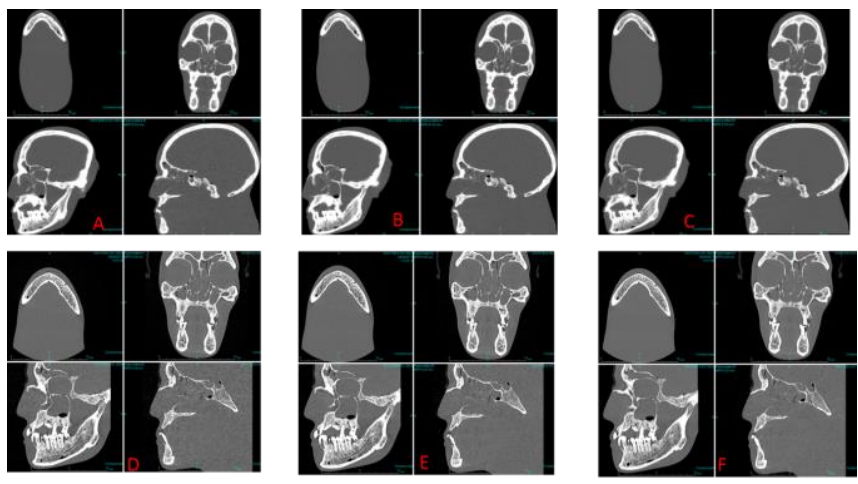

Figure 2. Sample of different exposure setting for GE and Toshiba 16 multislic CT. where images A,B, and $\mathrm{C}$ acquired with GE with exposure settings; $80 \mathrm{kVp}$ and $80 \mathrm{~mA}, 80 \mathrm{kVp}$ and $240 \mathrm{~mA}$ and $100 \mathrm{kVp}$ and $380 \mathrm{~mA}$ respectively. Where images $\mathrm{D}, \mathrm{E}$, and $\mathrm{F}$ acquired with Toshiba with exposure settings $100 \mathrm{kVp}$ and $100 \mathrm{~mA}, 100 \mathrm{kVp}$ and $120 \mathrm{~mA}$ and 120 $\mathrm{kVp}$ and $150 \mathrm{~mA}$ respectively.

Each image evaluation was based on the indication of the image pre-surgical planning stage of implant placement in the mandible; one side of the mandible was evaluated. In each image, 3 or 4 views has been presented. Therefore, the assessment of some anatomical landmarks may require several views. When ranking the visibility of the anatomical landmarks, no significant difference was established between anatomical landmarks. However, the lowest scoring will be allocated to mental and lingual foramina. The recorded subjective methods of evaluation of the image quality were then ranked from good, acceptable, and unacceptable image for implant placing. This ranking system was based on the equation below;

$$
C=\sum_{i=1}^{3} 1\left\{N_{i} \geq 5 \cap Q_{i} \geq 2\right\}
$$

Where $N_{i}$ is the number of the seven ALQ scored 4 or 5 for Observer $i$ and $Q_{i}$ is the score of OIQ given by Observer $i$. The function $1\{A\}$ is an indicator function which takes a value of 1 if the condition $A$ is met and 0 otherwise. Hence, $C$ can be interpreted as the number of observers scored at least 4 in five out of the seven anatomical landmarks quality (ALQ) and scored at least 2 at OIQ. The criteria is said to be met if $C$ is at least 2. Based on the values of $C$, clinical decisions can be made. In particular, the image quality is defined as "unacceptable" if the criteria is not met (i.e. $C \leq$ 1); "acceptable" if $C=2$ and "good" if $C=3$.

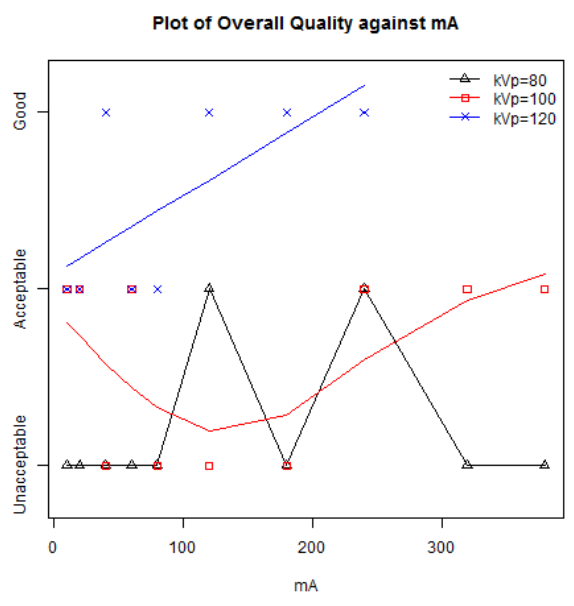

(A)

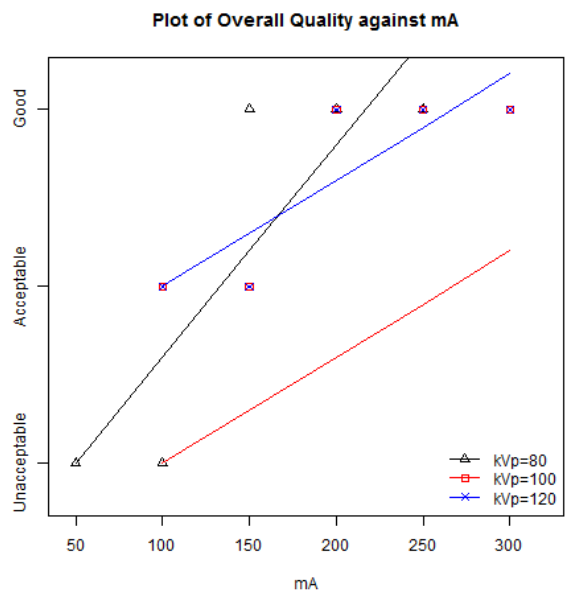

(B)

Figure 3. Image quality versus the tube current $(\mathrm{mA})$ with a cubic spline curve (smooth line), where the image (A) for GE device and image (B) for Toshiba device.

\section{Discussion}

Computed scanner numbers were analysed in an early Australian survey and it was identified that there were around 334 CT scanners, with the number largely increased by 2007 according to the United Nations Scientific Committee on the Effects of Atomic Radiation (UNSECAR) worldwide survey. Furthermore, according to this survey; japan was found to having the higher number of scanners relative to the population size ${ }^{15}$. A significant dose reduction can be achieved by cautiously considering individual characteristics, 
as well as each case's requirements ${ }^{16}$.

The justification practice means balancing the individual's or society's benefit with the inherent risk of radiation exposure (risk/benefit ratio). For optimization, the need to keep the dose levels "as low as reasonably achievable" (ALARA) is understood.

Exposure setting alteration can be achieved depending on the CT unit. Modifying these settings can result in a reduction of the radiation exposure dose; however, insufficient diagnostic image quality can be a side effect. Hence, a specific diagnostic indication should be asked for the possibility of exposure reduction ${ }^{15}$. The tube current represents the amount of electrons used to produce X-ray photons. Increasing the photons will enhance the image quality as there is a linear relation between $\mathrm{mA}$ settings and dose, thus reduction of tube voltage by half will result in a $50 \%$ reduction of dose ${ }^{17,18}$.

In this study, there were better image quality acquired with low dose image quality in comparison to the high dose especially with Toshiba device where the $\mathrm{kVp}$ alteration has not significantly affected the image quality with the image quality is always at least acceptable when the $\mathrm{mA}$ is higher than or equal to $150 \mathrm{~mA}$. On the other hand, with the GE device images have shown similar result For GE, when the $\mathrm{kV}$ is 120 , reduction of $\mathrm{mA}$ does not significantly affect the image quality (at least acceptable) which similar comparing to earlier studies which have shown that no significant difference was found in the visibility of mandibular anatomical landmarks ${ }^{18,19}$, while other studies have shown that the maxillofacial image qualities acquired by MSCT is comparable between low and high protocol of exposure parameters $^{20,21}$.

Both mental and lingual foramen were poorly acceptable clinically with low mA exposure settings, which may be as a result of increasing the image noise. However, the mandibular canal anatomical landmark has not been significantly affected with reduction of the tube energy which can explain that the increased noise can identify the outlines of the cortical regions around the mandibular canal ${ }^{22}$.

It is crucial to highlight that results in this present study are confined to the two CT systems (GE and Toshiba) that were used in this study and that caution should be exercised when other systems are taken into consideration. Limitation of this study includes the use of only two CT systems (GE and Toshiba 16 MSCT). In addition, values obtained for a phantom may not replicate the clinical situation. Finally, another limitation of the study was that the surrounding anatomical structures including the tongue and vertebra were absent, as standardizing the location of these structures in both modalities was rather burdensome.

\section{Conclusion}

In summary, utilising the GE MSCT in assessing the anatomical landmarks showed enhanced image quality as exposure tube current and voltages were increased. On the other hand, in the Toshiba MSCT, the image quality can be influenced through tube setting which is increased with high $\mathrm{mA}$ whilst the $\mathrm{kVp}$ has no significant modification on quality of image. Moreover, it was noted that reduction in the $\mathrm{mA}$ had negatively affected the quality of image of the mandibular implant placement, thus increasing the image noise.

\section{References}

(1) X. Liang, R. Jacobs, B. Hassan, L. Li, R. Pauwels, L. Corpas, P. C. Souza, W. Martens, M. Shahbazian, A. Alonso, and I. Lambrichts, "A comparative evaluation of Cone Beam Computed Tomography (CBCT) and Multi-Slice CT (MSCT) Part I. On subjective image quality," European journal of radiology 75 (2), 265-269, 2010).

(2) P. Pohlenz, M. Blessmann, L. Oesterhelweg, C. R. Habermann, P. G. Begemann, C. Schmidgunst, F. Blake, D. Schulze, K. Puschel, R. Schmelzle, and M. Heiland, "3D C-arm as an alternative modality to CT in postmortem imaging: technical feasibility," Forensic science international 175 (2-3), 134-139, 2008.

(3) T. Fuchs, M. Kachelriess, and W. A. Kalender, "Technical advances in multi-slice spiral CT," European journal of radiology 36 (2), 69-73, 2000.

(4) R. E. Jung, D. Schneider, J. Ganeles, D. Wismeijer, M. Zwahlen, C. H. Hammerle, and A. Tahmaseb, "Computer technology applications in surgical implant 
dentistry: a systematic review," The International journal of oral \& maxillofacial implants 24 Suppl, 92-109, 2009.

(5) B. Vandenberghe, S. Luchsinger, J. Hostens, E. Dhoore, and R. Jacobs, "The influence of exposure parameters on jawbone model accuracy using cone beam CT and multislice CT," Dento maxillo facial radiology 41 (6), 466-474, 2012.

(6) SS Halliburton, S Abbara, MY Chen, R Gentry, M Mahesh, GL Raff, LJ Shaw, J Hausleiter; Society of Cardiovascular Computed Tomography, " SCCT guidelines on radiation dose and dose-optimization strategies in cardiovascular CT," J Cardiovasc Comput Tomogr 5(4), 198-224, 2011.

(7) K. Horner, M. Islam, L. Flygare, K. Tsiklakis, and E. Whaites, "Basic principles for use of dental cone beam computed tomography: consensus guidelines of the European Academy of Dental and Maxillofacial Radiology," Dento maxillo facial radiology 38 (4), 187-195, 2009.

(8) A. Dawood, J. Brown, V. Sauret-Jackson, and S. Purkayastha, "Optimization of cone beam CT exposure for pre-surgical evaluation of the implant site," Dento maxillo facial radiology 41 (1), 70-74, 2012.

(9) A. Dawood, J. Brown, V. Sauret-Jackson, and S. Purkayastha, "Optimization of cone beam CT exposure for pre-surgical evaluation of the implant site," Dento maxillo facial radiology 41 (1), 70-74, 2012.

(10) K. F. Eckerman, "39th Lauriston S. Taylor Lecture: Dosimetry of Internal Emitters: Contribution of Radiation Protection Bodies and Radiological Events," Health physics 110 (2), 192-200, 2016.

(11) J. A. Hidalgo Rivas, K. Horner, B. Thiruvenkatachari, J. Davies, C. Theodorakou "Development of a lowdose protocol for cone beam CT examinations of the anterior maxilla in children," Br J Radiol 88 ( 1054), 2015.

(12) "The optimisation of radiological protection: broadening the process. ICRP publication 101. Approved by the Commission in September 2005," Annals of the ICRP 36 (3), 65, 71-104,2006.

(13) B. Casar, C. Lopes Mdo, A. Drljevic, E. Gershkevitsh, and C. Pesznyak, "Medical physics in Europe following recommendations of the International Atomic Energy Agency," Radiology and oncology 50 (1), 64-72, 2016.
(14) K. Matthews and PC Brennan, "Optimisation of X-ray examinations: General principles and an Irish perspective," Radiography 15 (3), 262-268, 2009.

(15) SC White, SM Mallya. "Update on the biological effects of ionizing radiation, relative dose factors and radiation hygiene". Aust Dent J.;57:2-8, 2012.

(16) J Sur, K Seki, H Kiozumi, K Nakajima, T Okano, "Effects of tube current on cone-beam computerized tomography image quality for presurgical implant planning in vitro", Oral Surg Oral Med Oral Pathol Oral Radiol Endod.; 110:29-33, 2010.

(17)XM Qu, G Li, JB Ludlow, ZY Zhang, XC Ma, 'Effective radiation dose of ProMax 3D cone-beam computerized tomography scanner with different dental protocols'. Oral Surg Oral Med Oral Pathol Oral Radiol Endod.;110:770-6. 2010.

(18) A Katsumata, A Hirukawa, S Okumura, M Naitoh, M Fujishita, E Ariji, et al. "Relationship between density variability and imaging volume size in cone-beam computerized tomographic scanning of the maxillofacial region: an in vitro study", Oral Surg Oral Med Oral Pathol Oral Radiol Endod; 107: 420-425, 2009.

(19)P Rustemeyer, U Streubuhr, J Suttmoeller, 'Low-dose dental computed tomography: significant dose reduction without loss of image quality', Acta Radiol.; 45:847-53, 2004.

(20) M Cohnen, H Fischer, J Hamacher, E Lins, R Kotter, U Modder, "CT of the head by use of reduced current and kilovoltage: relationship between image quality and dose reduction”, AJNR Am J Neuroradiol. ;21:1654-60, 2000.

(21) SA Sohaib, PD Peppercorn, JA Horrocks, MH Keene, GS Kenyon, RH Reznek, "The effect of decreasing mAs on image quality and patient dose in sinus CT". Br J Radiol.; 74:157-61, 2001.

(22) A Ekestubbe, K Grondahl, S Ekholm, PE Johansson, HG Grondahl, "Low-dose tomographic techniques for dental implant planning”, Int J Oral Maxillofac Implants.;11:650-9, 1996. 